Manuscript ID: 20-255-1-ED

“Development of a Smoke-free Homes Intervention for Parents: An Intervention Mapping Approach"

\title{
Response to reviewers' comments
}

We are extremely grateful to the reviewer for their time and effort in re-reviewing this manuscript following the changes that we made in relation to earlier reviewers' comments. We also appreciate the reviewers' positive comments regarding the adjustments already made to the paper, and we agree that some of their previous suggestions had not been completely addressed. We value their time taken to highlight the additional points that required attention or further elaboration. We provide detailed responses to each point in the document below and have used tracked changes to highlight these changes in the manuscript.

\section{Reviewer A - Overall:}

Figure 1 gives a good overview of the steps included in the Intervention Mapping approach and the tasks that are included in each step (as indicated with the bullets). Since the paper contains a lot of information and is quite long, it might be good to explicitly include the different task per step. For instance, for step 1, it is good to first have the establishment of a planning group as the first task. After this, health, quality of life, behaviour and the environment can be dealt with when discussing the review, etc. This will help to reader to follow the systematic approach.

\section{Response:}

We have now revised Figure 1 to include an outline of the IM steps AND the different tasks that were undertaken for each step. We agree that this should help the reader to better follow our approach, and we thank you for your suggestion.

It is very good to see that a lot of material has been uploaded as supplementary files. However, I miss clear references to these materials in the text. It might be good to do this, in order to guide the reader to and through all supplementary materials.

\section{Response:}

Thank you for highlighting this. We have now made clear reference to all supplementary materials in the text as you've suggested.

\section{Step 1:}

During my first review I have suggested to create a Logic Model of the Problem, as this is the core of step 1 (which will then serve as the basis for the other steps). The response of the authors was the following: "We have considered your suggestion to create a logic model of the problem, and we believe that for more complex needs assessments this would be a valuable means of adding clarity. However, we feel having expanded our review, that the factors and determinants involved in creating a smoke-free home should be clear to the reader." In response to this, I want to mention that expanding on the literature review is very useful. However, this will make the need for an overview of the outcomes of these reviews even more urgent. At this point, the reader had to make considerable effort to get a grasp of the outcomes of this review ( = what is the health problem, what are the behaviours of the target group and the environmental agents involved and what are the determinants related to these behaviours), so adding a summary of all information in the form of a 
Logic Model of the Problem will therefore be an even more valuable contribution. This summary is important for the reader to fully grasp the problem, as this will serve as the foundation for the following steps.

Response: Thank you for clarifying again the rationale for including a Logic Model of the Problem, which we have now developed and included as Figure 2. We agree that this should help the reader to fully grasp the issues at hand.

Step 2:

In my comments based on the previous version I had suggested to:

- $\quad$ include a Logic Model of Change. The authors have responded by saying "that a logic model would replicate the information provided already in the matrices. Perhaps if this were a more complicated set of issues then the addition of a logic model would be most useful." Even though I still feel that adding such a model is a very simply, but extremely clarifying step to take, I understand the authors.

However, at this point, I find it difficult to see the link between step 1 (Logic Model of the Problem) and step 2 (The Logic Model of Change). How are the proposed outcomes (SMART outcomes, POs and $\mathrm{COs}$ ) related to the description of the problem indicated in step 1? Adding both models can help the reader to clearly see the description of the problem, to see the description of the solution and, most importantly, see the so-called 'flip' that is conducted in step 2.

Response: We now understand the rationale for including both models, and have developed and included a Logic Model of Change as Figure 3. Thank you for your helpful suggestion.

I have some difficulties with distinguishing between the outcome 1 and 2 . The authors clearly state that "our first matrix is focused on parents (mothers, fathers, step-parents)" to not smoke indoor. All POs are aligned to this aim. However, if both parents are included in outcome 1 , who will they then target in outcome 2? If find it difficult to assess the relevance of this outcome. In other words, how is this outcome (and thus developing an intervention to achieve this) a solution to the problem described in step 1 ?

Response: Thank you for raising this point, which we agree required clarification in the text. We have added text on page 6 and 7 to address this. Outcome 1 does include all participating parents, including those actively taking part in the intervention, and their partners and other adult household members who smoke if applicable. Table (Matrix) 1 is therefore focused on all parents/carers involved in the intervention. Outcome $\mathbf{2}$ focuses on the act of successfully engaging partners/other household members in the intervention, and the accompanying Table (Matrix) 2 outlines the steps required for parents to effectively extend intervention delivery in this way. These differences are also highlighted within our Logic Model of Change. We hope that this now clarifies the distinction between outcome 1 and 2 .

- $\quad$ Add a clear rationale provided for using the Self-Determination Theory (SDT). The authors have responded by saying that "we should have made it clearer that the determinants and change objectives in our matrices were developed on the basis of our literature review findings, the Self Determination Theory and Control Theory". Even though this topic is addressed in the text and the link with the literature is indeed explicated, it might be good to reflect on how the content of the SDT is translated into the POs and the determinants. For instance, how are the POs linked to the SDT, or which of the selected determinants are linked to the SDT? 
- Reflect on the how determinants were selected (which theoretical and/or empirical evidence was used here?). The authors have responded with saying "we have stopped short of setting out precisely which theoretical and/or empirical evidence was used to generate each one. However, we do take your point and we have therefore made it more explicit that all the POs, determinants and change objectives were derived either from existing literature, our review work, or based on Self Determination Theory and Control Theory as our main theories." This is very good to hear. I also understand, and know from experience, that this is a step process that can be time and effort consuming. I would advise the authors though to also briefly reflect not only on the fact that this was done, but also how this was done. So, how are the POs and the selected determinants linked to these theories? And why are these theories especially useful when it comes to the behaviours depicted in the main outcomes?

Response: Thank you for these helpful suggestions. We have now incorporated more detailed text on pages 7,8 and 13 which explains how our performance objectives and determinants link to our selected theories and the empirical evidence, and why the theories we selected are especially useful in relation to the behaviour changes required to create a smoke-free home. 